\title{
A NOTE ON NEIGHBOURHOODS OF UNIVALENT FUNCTIONS
}

\author{
RICHARD FOURNIER
}

\begin{abstract}
Using a notion of neighbourhood of analytic functions due to Stephan Ruscheweyh we examine conditions under which neighbourhoods of a certain class of convex functions are included in a class of starlike functions.
\end{abstract}

Introduction. Let $A$ denote the class of analytic functions $f$ in the unit disk $E$ : $\{z|| z \mid<1\}$ with $f(0)=0, f^{\prime}(0)=1$. For $f(z): z+\sum_{k=2}^{\infty} a_{k} z^{k} \in A$ and $\delta \geqslant 0$ Ruscheweyh has defined the neighbourhood $N_{\delta}(f)$ as follows:

$$
N_{\delta}(f):=\left\{g(z):=z+\sum_{k=2}^{\infty} b_{k} z^{k}\left|\sum_{k=2}^{\infty} k\right| a_{k}-b_{k} \mid \leqslant \delta\right\} .
$$

He has shown in [1] among other results that if $f(z):=z+\sum_{k=n+1}^{\infty} a_{k} z^{k} \in C$ the following result is true:

$$
N_{\delta_{n}}(f) \subset S^{*}, \quad \delta_{n}=2^{-2 / n},
$$

where $C\left(S^{*}\right)$ denotes the class of normalized convex (starlike) univalent functions in $A$. He also asked if a similar result would hold if we replace $S^{*}$ by the class

$$
T:=\left\{g \in S^{*}|| z \frac{g^{\prime}(z)}{g(z)}-1 \mid<1, z \in E\right\}
$$

and $C$ by the class

$$
\tilde{T}:=\left\{g \in C|| z \frac{g^{\prime \prime}(z)}{g^{\prime}(z)} \mid<1, z \in E\right\}
$$

We prove

THEOREM 1. Let $f(z):=z+\sum_{k=n+1}^{\infty} a_{k} z^{k} \in \tilde{T}$. Then $N_{\delta_{n}}(f) \subset T, \delta_{n}=e^{-1 / n}$.

Let $S_{\alpha}^{*}(0 \leqslant \alpha<1)$ denotes the class $\left\{g \in S^{*} \mid \operatorname{Re}\left[z\left(g^{\prime}(z) / g(z)\right)\right]>\alpha, z \in E\right\}$. An analogue of this class with respect to $T$ is the class

$$
T_{r}:=\left\{g \in T|| z \frac{g^{\prime}(z)}{g(z)}-1 \mid<r, z \in E\right\}, \quad 0<r \leqslant 1 .
$$

Received by the editors March 23, 1982.

1980 Mathematics Subject Classification. Primary 30C45: Secondary 30C75.

Key words and phrases. Univalent starlike and convex functions, neighbourhoods of analytic functions, Hadamard product. 
In [1] Ruscheweyh has shown that for no $\alpha \in[0,1)$ is there a positive $\delta$ such that $N_{\delta}\left(S_{\alpha}^{*}\right) \subset S^{*}$. For the class $T_{r}$ the situation is quite different as shown by

THEOREM 2. Let $g(z):=z+\sum_{k=n+1}^{\infty} a_{k} z^{k} \in T_{r}, 0 \leqslant r<1$. Then $N_{\delta_{n}}(g) \subset T$, $\delta_{n}=e^{-r / n}(1-r)$.

The boundaries of $\{w \in \hat{\mathbf{C}} \mid \operatorname{Re}[w]>0\}$ and of $\{w \in \hat{\mathbf{C}} \mid \operatorname{Re}[w]>\alpha\}$ are not disjoint whereas those of $\{w \in \hat{\mathbf{C}}|| w-1 \mid<1\}$ and of $\{w \in \hat{\mathbf{C}}|| w-1 \mid<r\}$ are; this is one of the reasons for the difference between the two situations. Nevertheless Theorem 2 is still interesting since the value for $\delta_{n}$ is best possible.

Concerning this question of boundaries we can prove

THEOREM 3. Let $f \in T$ and $D:=\left\{z f^{\prime}(z) / f(z) \mid z \in E\right\}$ be such that there is $w \in \bar{D}$ with $|w-1|=1$. Then for no $\delta>0$ we have $N_{\delta}(f) \subset T$.

It should be noted that no similar result holds if the class $T$ is replaced by the class $S^{*}$; in fact for $f(z)=z /(1-z) \in C \subset S^{*}$ we have $N_{1 / 4}(f) \subset S^{*}$ even though the region $D=\left\{w \in \mathbf{C} \mid \operatorname{Re}[w]>\frac{1}{2}\right\}$ is such that the point at infinity belongs to both $\bar{D}$ and $\{w \in \hat{\mathbf{C}} \mid \operatorname{Re}[w] \geqslant 0\}$.

Proof of Theorem 1. It was established in [2] that for $f(z):=z+\sum_{k=2}^{\infty} a_{k} z^{k} \in T$ we have the estimate $|z| e^{-|z|} \leqslant|f(z)| \leqslant|z| e^{|z|}$; using the same method it is very easy to show that for $f(z):=z+\sum_{k=n+1}^{\infty} a_{k} z^{k} \in T$ the estimate

$$
|z| e^{-|z|^{n} / n} \leqslant|f(z)| \leqslant|z| e^{|z|^{n} / n}
$$

is true and sharp as seen from the function $f(z):=z e^{z^{n} / n}$. We also remark that $f(z) \in \tilde{T} \Leftrightarrow z f^{\prime}(z) \in T$ so that we obtain for $f(z):=z+\sum_{k=n+1}^{\infty} a_{k} z^{k} \in \tilde{T}$ for the following estimate

$$
e^{-|z|^{n} / n} \leqslant\left|f^{\prime}(z)\right| \leqslant e^{|z|^{n} / n}
$$

and the sharpness is established by looking at the function $f(z):=\int_{0}^{z} e^{u^{n} / n} d u$.

We also remark the following: a function $g(z) \in A$ belongs to the class $T$ iff for every $\theta \in[0,2 \pi)$ we have

$$
z \frac{g^{\prime}(z)}{g(z)}-1 \neq e^{i \theta}, \quad z \in E
$$

that is

$$
\frac{1}{z}\left(\left(\frac{z /(1-z)^{2}-\left(1+e^{i \theta}\right) z /(1-z)}{-e^{i \theta}}\right) * g(z)\right) \neq 0, \quad \theta \in[0,2 \pi), z \in E,
$$

where * denotes the Hadamard product of two functions. Since

$$
-e^{i \theta} h_{\theta}(z):=\frac{z}{(1-z)^{2}}-\left(1+e^{i \theta}\right) \frac{z}{1-z}=-e^{i \theta} z+\sum_{n=2}^{\infty}\left(n-1-e^{i \theta}\right) z^{n}
$$


where $\left|n-1-e^{i \theta}\right| \leqslant n$ it is clear from the results in [1] that a sufficient condition in order that $N_{\delta}(f) \subset T$ may hold for some function $f$ in $A$ is that

$$
\left|\frac{h_{\theta}(z) * f(z)}{z}\right| \geqslant \delta, \quad z \in E, \theta \in[0,2 \pi) .
$$

Now let $f(z):=z+\sum_{k=n+1}^{\infty} a_{k} z^{k} \in \tilde{T}$. We have

$$
\begin{aligned}
f(z) * h_{\theta}(z) & =\frac{z f^{\prime}(z)-\left(1+e^{i \theta}\right) f(z)}{-e^{i \theta}}, \\
\frac{\left(f(z) * h_{\theta}(z)\right)^{\prime}}{f^{\prime}(z)} & =1-e^{-i \theta} z \frac{f^{\prime \prime}(z)}{f^{\prime}(z)}
\end{aligned}
$$

with $\operatorname{Re}\left[1-e^{i \theta} z f^{\prime \prime}(z) / f^{\prime}(z)\right] \geqslant 1-\left|z f^{\prime \prime}(z) / f^{\prime}(z)\right|>0$. This shows, since $f \in \tilde{T} \subset$ $C$, that the functions $h_{\theta}(z) * f(z)$ are close-to-convex univalent. We also get the estimate

$$
\left|\left(f(z) * h_{\theta}(z)\right)^{\prime}\right| \geqslant\left|f^{\prime}(z)\right|\left(1-\left|z \frac{f^{\prime \prime}(z)}{f^{\prime}(z)}\right|\right) \geqslant e^{-|z|^{\prime \prime} / n}\left(1-|z|^{n}\right)
$$

using (2) and Schwarz lemma. Since the functions $h_{\theta}(z) * f(z)$ are univalent we can integrate the last estimate to obtain

$$
\left|f(z) * h_{\theta}(z)\right| \geqslant \int_{0}^{|z|} e^{-u^{n} / n}\left(1-u^{n}\right) d u=|z| e^{-|z|^{n} / n}
$$

so that according to (3), $N_{\delta_{n}}(f) \subset T$ for $\delta_{n}=e^{-1 / n}$. The sharpness of the result is seen from the function $f(z):=\int_{0}^{z} e^{u^{n} / n} d u$; in fact $g(z):=f(z)+\delta_{n} z^{n+1} /(n+1)$ $\in N_{\delta_{n}}(f)$ and $g^{\prime}(z)=f^{\prime}(z)+\delta_{n} z^{n}=0$ if $z^{n}=-1$. This completes the proof of Theorem 1 .

Proof of Theorem 2. The proof of Theorem 2 is more direct. We first remark that from the definition of $T_{r}$ we have

$$
g(z) \in T_{r} \Leftrightarrow g(z)=z\left(\frac{g_{1}(z)}{z}\right)^{r} \text { for some function } g_{1} \in T
$$

so that if $g(z):=z+\sum_{k=n+1}^{\infty} a_{k} z^{k} \in T_{r}$ we get from (1) and Schwarz lemma that

$$
\begin{aligned}
& e^{-r|z|^{n} / n} \leqslant\left|\frac{g(z)}{z}\right| \leqslant e^{r|z|^{n} / n}, \\
& \left|z \frac{g^{\prime}(z)}{g(z)}\right| \leqslant r|z|^{n} .
\end{aligned}
$$

Now let $0 \leqslant \theta<2 \pi$; we have, according to (4) and (5), for $z \in E$,

$$
\begin{aligned}
\left|\frac{g(z) * h_{\theta}(z)}{z}\right| & =\left|g^{\prime}(z)-\left(1+e^{i \theta}\right) \frac{g(z)}{z}\right| \geqslant\left|\frac{g(z)}{z}\right|\left(1-\left|z \frac{g^{\prime}(z)}{g(z)}-1\right|\right) \\
& \geqslant e^{-r|z|^{n} / n}\left(1-r|z|^{n}\right)
\end{aligned}
$$

from which it follows, according to (3), that $N_{\delta_{n}}(g) \subset T$ for $\delta_{n}=(1-r) e^{-r / n}$. The sharpness of the result is. seen from the function $g(z):=z e^{r z^{n} / n}$; in fact, 
$f(z):=g(z)+\delta_{n} z^{n+1} /(n+1) \in N_{\delta_{n}}(g)$ and $f^{\prime}(z)=0$ if $z^{n}=-1$. This completes the proof of Theorem 2 .

Proof of Theorem 3. Let $h_{\theta}(z)$ be defined as before. Since

$$
\frac{f(z) * h_{\theta}(z)}{z}=-e^{i \theta} \frac{f(z)}{z}\left(\left(z \frac{f^{\prime}(z)}{f(z)}-1\right)-e^{i \theta}\right)
$$

it is clear from the hypothesis on $D$, that, $|f(z) / z|$ being bounded in $E$,

$$
\inf \left|\frac{f(z) * h_{\theta}(z)}{z}\right|=0
$$

where the inf is taken over all $z \in E, \theta \in[0,2 \pi)$.

We now proceed to show Theorem 3 following an idea due to Ruscheweyh [1]. Let $\delta>0$ and $n$ some integer $>2$. Choose a point $z_{0} \in E$ and $\theta \in[0,2 \pi)$ such that for $\mu:=\left(f * h_{\theta}\left(z_{0}\right)\right) / z_{0}^{n}$ we have

$$
|\mu|=\left|\frac{f * h_{\theta}\left(z_{0}\right)}{z_{0}^{n}}\right|<\delta\left(\frac{n-2}{n}\right) .
$$

This is always possible because of (6) and the fact that the function $f(z) * h_{\theta}(z), f$ being in the class $T$, is nonvanishing for $z \neq 0$. We then define the function $g(z):=f(z)-\mu z^{n} / a_{n}$ where $a_{n}:=h_{\theta}^{(n)}(0) / n !=\left(n-1-e^{i \theta}\right) /-e^{i \theta}$; it is clear that $\left|a_{n}\right| \geqslant n-2$ so that $n\left|\mu / a_{n}\right| \leqslant n|\mu| /(n-2)<\delta$ and $g \in N_{\delta}(f)$; but on the other side we have

$$
\frac{g * h_{\theta}\left(z_{0}\right)}{z_{0}}=\frac{f * h_{\theta}\left(z_{0}\right)}{z_{0}}-\mu z_{0}^{n-1}=0
$$

which shows that $g \notin T$. This completes the proof of Theorem 3 .

\section{REFERENCES}

1. St. Ruscheweyh, Neighbourhoods of univalent functions, Proc. Amer. Math. Soc. 81 (1981), $521-527$.

2. Ram Singh, On a class of star-like functions, Compositio Math. 19 (1968), 78-82.

Department of Mathematics, University of Montreal, Montreal, Quebec, Canada H3C 3J7 